\title{
USING THE LOAD-VELOCITY RELATIONSHIP FOR 1RM PREDICTION
}

\author{
Boris Jidovtseff,,${ }^{1,2}$ Nigel K. Harris, ${ }^{2}$ Jean-Michel Crielaard, ${ }^{1}$ and John B. Cronin ${ }^{2}$ \\ ${ }^{1}$ University of Liège, Department of Sports Sciences, Service of Training and Assessment of Physical Fitness, Liège, Belgium; and \\ ${ }^{2}$ Institute of Sport and Recreation Research New Zealand, Auckland University of Technology, Auckland, New Zealand
}

\begin{abstract}
Jidovtseff, B, Harris, NK, Crielaard, J-M, and Cronin, JB. Using the load-velocity relationship for 1 RM prediction. J Strength Cond Res 24(x): 000-000, 2009-The purpose of this study was to investigate the ability of the load-velocity relationship to accurately predict a bench press 1 repetition maximum (1 RM). Data from 3 different bench press studies $(n=112)$ that incorporated both $1 \mathrm{RM}$ assessment and submaximal load-velocity profiling were analyzed. Individual regression analysis was performed to determine the theoretical load at zero velocity (LD0). Data from each of the 3 studies were analyzed separately and also presented as overall group mean. Thereafter, correlation analysis provided quantification of the relationships between 1 RM and LD0. Practically perfect correlations $(r=\sim 0.95)$ were observed in our samples, confirming the ability of the loadvelocity profile to accurately predict bench press $1 \mathrm{RM}$.
\end{abstract}

KEYWORDS : maximal strength ; isoinertial ; estimation

\section{INTRODUCTION}

Determination of an individual's maximal strength or 1 repetition maximum (1RM) is considered by some authors to be problematic for populations such as the young (18) and the aged (23). As a result, different authors have reported methods that allow the estimation of 1RM from performing submaximal repetitions to failure $(1,4,13,21,22)$. The repetitions to failure method has been widely investigated, the consensus being that the accuracy of these estimations depends on several parameters such as the number of repetitions, type of exercise, training background, and population used $(3,5,8,13,21)$. However, all these methods are based on the forceendurance relationship and no author to our knowledge has proposed estimation of 1RM from the load-velocity relationship. Given the increasing use of dynamometers such as accelerometers and/or linear position transducers, it may be that maximal strength can be predicted from the load-velocity relationship within individuals. The aim of this study was to first examine the relationship between 1RM and the load-velocity profile and subsequently determine 1RM prediction equations from that profile.

\section{METHODS}

\section{Experimental Approach to the Problem}

Theoretically, the load-velocity relationship could be used to estimate maximal strength such as 1RM. The data of 3 studies that determined both the 1RM and load-velocity relationship for the bench press were used for analysis. The measurement of average velocity (AV) at 3 or 4 increasing loads in these studies determined, for each subject, the best fit regression line and associated parameters such as slope and intercept point on the $\mathrm{Y}$ axis that could be used for 1RM estimation.

\section{Subjects}

A total of 112 subjects (90 males, 22 females) from 3 previous studies $(14,15,17)$ were involved in this analysis. Table 1 summarizes the subject characteristics. All subjects were involved in recreational activities and were free from any injuries at the time of each investigation. Study protocols were approved by the University of Liege Ethical Committee, and all subjects signed an informed consent form prior to each study. 


\section{Equipment}

A linear position transducer (Celesco Transducer Products, Inc., model PT5DC, Chatsworth, California, USA) was used to determine displacement-time data from which velocity during a bench press (BP) exercise performed on a Smith machine (Multipower M433, Salter S.A., Barcelona, Spain) was derived. The equipment used in the present study has been previously described (16).

\section{Procedures}

All subjects involved in each of the studies underwent the same assessment procedures. The first session was dedicated to familiarization with the bench press exercise, determination of the standardized position, and 1RM assessment according to the standard methods established by Kraemer and Fry (19). The bench press exercise was a concentric-only movement and started with the barbell $3 \mathrm{~cm}$ above the subject's nipple line. Hand position on the bar was defined as the distance measured between elbows at 90 degrees of shoulder abduction. During the whole movement, subjects had to keep the back on the bench and their hips flexed at 90 degrees. During the second session, which occurred approximately 1 week after the initial 1RM assessment, all subjects were tested at 3 or 4 increasing bench press loads, as presented in Table 1. The number of trials performed at each load was as follows: 4 trials at 30, 35, and 40\% 1RM; 3 trials at 50, 60, and 70\% 1RM; and 2 trials at 80, 90, and 95\% 1RM. For each load, subjects were instructed to lift the barbell as fast as possible, but they were not allowed to release the bar. The best trial was selected for analysis in terms of the highest velocity value. Average velocity (AV) was measured for the entire concentric phase of each bench press. Intertriai and intersession reliability of this parameter has been demonstrated to be very good during concentric bench press assessment (coefficient of variation $[\mathrm{CV}]<7 \%)(16)$.

TABLE 1. Physical characteristics (mean $\pm S D$ ) for each group and relative loads used for the force-velocity relationship establishment.

\begin{tabular}{lcccccc}
\hline Study & $N$ & Gender & Age (years) & Height $(\mathrm{m})$ & Weight $(\mathrm{kg})$ & Percent 1 RM assessed (\%) \\
\hline S1 & 35 & M & $23 \pm 2$ & $1.80 \pm 0.05$ & $77 \pm 8$ & $35,50,70$, and 90 \\
S2 & 55 & M & $24 \pm 4$ & $1.79 \pm 0.05$ & $73 \pm 9$ & 40,60, and 80 \\
S3 & 22 & F & $21 \pm 2$ & $1.67 \pm 0.07$ & $61 \pm 9$ & $30,50,70$, and 95 \\
Total & 112 & M+F & $23 \pm 4$ & $1.77 \pm 0.11$ & $72 \pm 14$ & - \\
\hline
\end{tabular}

$1 \mathrm{RM}=1$ repetition maximum.

\section{Data Analysis}

Data were sampled at $1,000 \mathrm{~Hz}$ via an analogue-to-digital interface (PCMCIA DAQCard, 6024E, National Instrument, Austin, Texas, USA). Labview software (National Instrument) enabled data analysis, storage of information, and instantaneous presentation of results. Velocity was derived from the displacement signal with the 2nd Order Central method. A low-pass filter was used to smooth velocity data with a low cut-off of $17 \mathrm{~Hz}$.

\section{Statistical Analyses}

Means and standard deviations are used throughout as measures of centrality and spread of data. For each subject the best-fit regression line was plotted through the known load (X) and AV (Y) values using Excel software (Microsoft, Redmond, Washington, USA). From this linear regression, the slope (SLP), theoretical AV at $0 \mathrm{~kg}$ (AVO), and theoretical load at $0 \mathrm{~m} / \mathrm{s}^{-1}$ (LD0) were calculated. Mean values and standard deviation of these data were then used to describe the change in average velocity with increasing relative load. The accuracy of the prediction formula was calculated using the standard error of estimate as follows:

$$
\mathrm{SEE}=\sqrt{\frac{\sum(\mathrm{Y}-\mathrm{Y})^{2}}{\mathrm{~N}}}
$$

The magnitudes of the relationships between actual and predicted 1RM were interpreted using Pearson correlation coefficients, described as trivial (0.0-0.1), low (0.1-0.3), moderate (0.3-0.5), high (0.5-0.7), very high $(0.7-0.9)$, or practically perfect $(0.9-1.0)(6,12)$. An inference about the true (large-sample) value of a correlation 
was based on uncertainty in its magnitude (2); if the $90 \%$ confidence limits overlapped substantial positive and negative values, the magnitude was deemed unclear; otherwise the magnitude was deemed to be the observed magnitude. The confidence interval was derived via the Fisher $\mathrm{z}$ transformation (11); for trivial-small correlations the confidence limits were $\sim \pm 0.15$. Thus the power of this study was such that only correlations greater than $>0.25$ or $<-0.25$ were considered clear. Given the bipolar nature of male and female values that artificially inflate correlation coefficients, the strength of the relationships was expressed for each sample.

Figure 1. Theoretical AV-relative load (\% 1 repetition maximum) relationship according to mean values observed in the whole population. The thick line represents the mean tendency; thin lines represent $\pm 1 S D . A V=$ average velocity; $L D O=$ load at zero velocity.

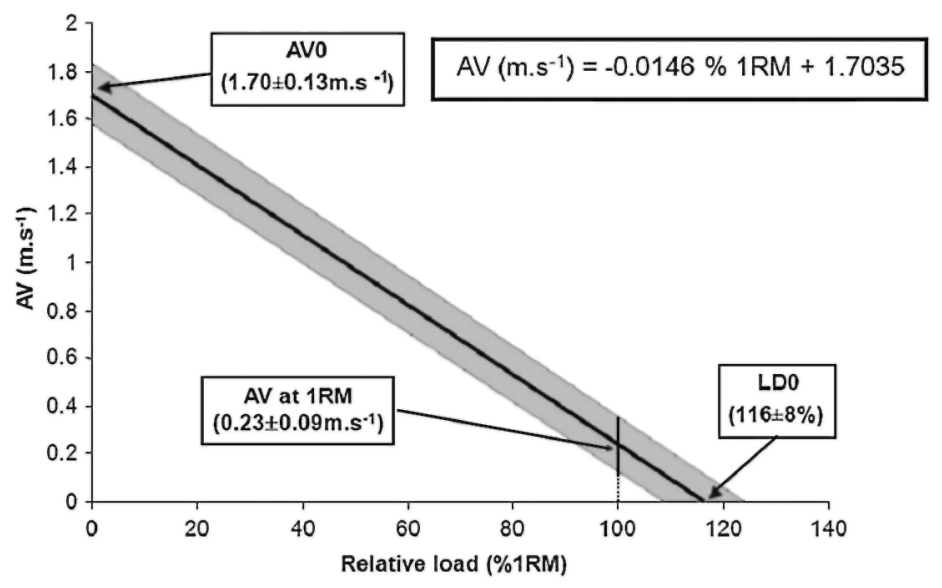

Figure 2. Relationship between actual 1 repetition maximum and load at zero velocity (LDO) of all 3 study groups.

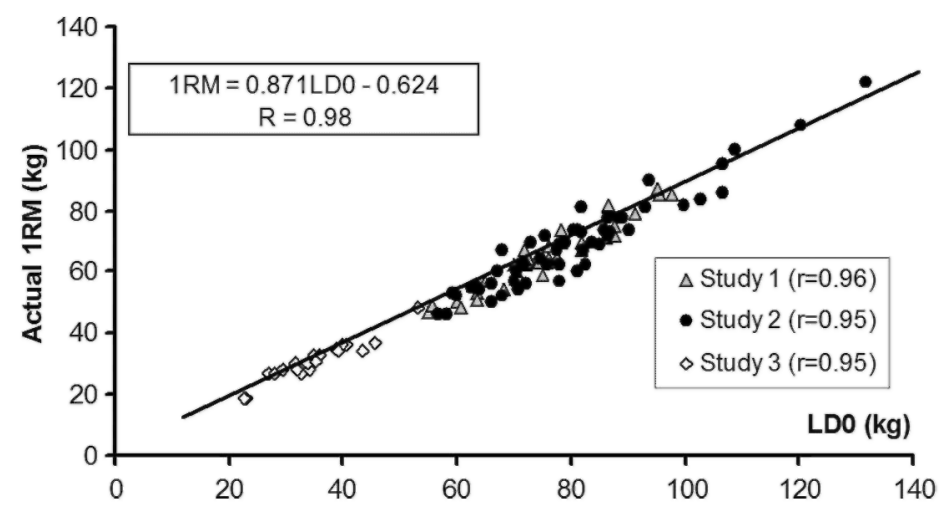

\section{RESULTS}

For the whole population, bench press $1 \mathrm{RM}$ was $60 \pm 19 \mathrm{~kg}$ Mean and standard deviation for the SLP, AVO, and LD0 were, respectively: $-0.028 \pm 0.012 \mathrm{~m} \cdot \mathrm{s}^{-1} \cdot \mathrm{kg}^{-1}, 1.70 \pm 0.13 \mathrm{~m} \cdot \mathrm{s}^{-1}$, and $70 \pm 22 \mathrm{~kg}$.

LD0 corresponded to $116 \pm 8 \%$ of the $1 \mathrm{RM}$. Figure 1 illustrates the mean linear regression between AV and relative load. AV at $1 \mathrm{RM}$ was $0.23 \pm 0.09 \mathrm{~m} \cdot \mathrm{s}^{-1}$.

The correlations between 1RM and load at zero velocity are illustrated in Figure 2. The correlations were practically perfect for study $1(r=0.96)$, study $2(r=0.95)$, and study $3(r=0.95)$. The cumulative correlation for the entire sample was $r=0.98$. 


\section{DISCUSSION}

Our results confirmed that the load-velocity relationship can be used to estimate maximal strength performance (i.e., 1RM). We chose to analyze the AV rather than the peak velocity for 2 reasons. First, AV better represents the ability of the subject to move the load throughout the entire concentric phase. Second, as previously demonstrated in concentric bench press assessment (16), AV decreases linearly with increasing load, making mathematical analysis easier.

The analysis of the AV-relative load relationship revealed that the movement velocity associated with $1 \mathrm{RM}$ is $0.23 \pm 0.09 \mathrm{~m} \cdot \mathrm{s}^{-1}$ and LD0 corresponds to $116 \pm 8 \%$ of the $1 \mathrm{RM}$. The results support findings that maximum isometric force is greater than the maximum concentric force (10) as per the force-velocity relationship of muscle. In addition, it is well known that the 1RM corresponds to the highest load that can be lifted for the whole concentric phase and therefore depends on the strength developed in the weakest position. In the bench press this position, also called the "sticking region," occurs at approximately 10 to $15 \mathrm{~cm}$ above the chest $(9,20)$. The assessment of velocity or acceleration at that position may be interesting because the bar should be close to zero when a subject reaches his or her 1RM.

A practically perfect correlation between LD0 and the 1RM $(r=0.98)$ was found, providing evidence that the load-velocity relationship may be used to estimate 1RM using the following equation:

$$
1 \mathrm{RM}=(0.871 \mathrm{LD} 0)-0.624(\text { equation } 1)
$$

This formula offers a reasonable prediction of the 1RM with a standard error of estimate (SEE) of $4 \mathrm{~kg}(7 \%)$. This approach of using the load-velocity relationship to predict maximal isoinertial strength appears to be at least as accurate as the repetitions to failure method. According to the review of Pereira and Gomes (22), predicting 1RM from maximum repetition tests results in fairly good correlations $(r>0.68)$. However, the number of repetitions and the relative load used appear critical to ensure accuracy. Prediction with 10 or fewer repetitions has resulted in correlations of greater than 0.9 between the predicted and actual IRMs and SEE $<10 \mathrm{~kg}(22)$. Cosgrove and Mayhew (7) reported that light-load endurance tests lead to less accurate prediction of 1RM. Hence, it is generally recommended that heavier (75\% 1RM and higher) loads be used for the most accurate prediction of 1RM from submaximal load testing (24). In our study, even when we estimated the 1RM with 2 submaximal loads (30-35\% and 70\% for S1 and S3; 40 and $60 \%$ for S2), the correlation between LD0 and 1RM was practically perfect $(r=0.96)$ and the SEE did not exceed $5 \mathrm{~kg}$.

It appears that the prediction of bench press $1 \mathrm{RM}$ from the load-velocity relationship is just as accurate as the repetition to failure method; however, the former method can use lighter loads and does not require lifting to failure, which may be suitable for a broader range of populations. Clearly, further research is needed to explore the ability of the load-velocity relationship to predict 1RM in other exercises. Moreover, this method appears of great interest because it can be used concurrently for 1RM prediction and velocity/power profiling at submaximal loads. Such an approach is an intuitively appealing strategy for athlete assessment and monitoring. The major limitation of this method is the necessity of inertial dynamometers such as linear position transducers or accelerometers, however, with time we believe the use of such technology will be commonplace in most weight training facilities.

\section{Practical Applications}

We recommend that strength and conditioning practitioners who want to estimate bench press 1RM from the AV-load relationship need to measure AV at 3 or 4 increasing loads, starting with a light load. The load increment may be customized. It is unnecessary to use very heavy loads, but the decrease in AV between the lightest and heaviest loads should reach at least $0.5 \mathrm{~m} \cdot \mathrm{s}^{-1}$ to significantly cover the load-velocity relationship. The consequent linear regression allows the calculation of LD0 and subsequently the estimation of $1 \mathrm{RM}$ by using equation 1 . To get the subject's best performance, it is recommended that 3 to 5 separate trials be performed at each load and a full recovery period be allowed between trials given.

\section{ACKNOWLEDGMENT}

No funding was received for this research.

\section{REFERENCES}


1. Abadie, BR and Wentworth, MC. Prediction of one repetition maximal strength from a 5-10 repetition submaximal strength test in college-aged females. J Exerc Physiol 4: 1-6, 2000.

2. Batterham, AM and Hopkins, WG. Making meaningful inferences about magnitudes, hit J Sports Physiol Perf 1: 50-57, 2006.

3. Becque, M and Pick, J. Validity of predicting 1RM and \%1RM in weight-trained and untrained males. Med Sci Sports Exerc 27: S10, 1995.

4. Berger, RA. Determination of the resistance load for 1RM and 10RM. J Assoc Phys Mental Rehab 15: 108-110, 1961.

5. Braith, R, Graves, J, Leggett, S, and Pollock, M. Effect of training on the relationship between maximal and submaximal strength. Med Sci Sports Exerc 25: 132-138, 1993.

6. Cohen, J. Statistical PowerAnalysis for the Behavioural Sciences. (2nd éd.). Hillsdale, NJ: Lawrence Erlbaum, 1988.

7. Cosgrove, L and Mayhew, JL. A modified YMCA bench press test to predict strength in adult women. IAHPERD J 30: 25-26, 1997.

8. Dohoney, P, Chromiak, JA, Lemire, D, Abadie, B, and Kovacs, C. Prediction of one repetition maximum (1-RM) strength from a 4-6 RM and a 7-10 RM submaximal strength test in healthy young adult males. J Exerc Physiol S: 54-59, 2002.

9. Elliott, BC, Wilson, GJ, and Kerr, GK A biomechanical analysis of the sticking region in the bench press. Med Sci Sports Exerc 21: 450462,1989

10. Enoka, RM. Neuromechanics of Human Movement. Champaign, IL: Human Kinetics, 2002.

11. Fisher, RA. On the probable error of a coefficient of correlation deduced from a small sample. Metron 1: 3-32, 1921.

12. Hopkins, WG. A scale of magnitudes for effect statistics. Available at: http://www.sportsci.org/resource/stats/index.html. Accessed 2002.

13. Horvat, M, Ramsey, V, Franklin, C, Gavin, C, Palumbo, T, and Glass, LA. A method for predicting maximal strength in collegiate women athletes. J Strength Cond Res 17: 324-328, 2003.

14. Jidovtseff B, Cordonnier, C, Vandermeulen, M, and Crielaard, JM. Gender differences in force-power-velocity relationships. Isokinetics Exerc Sci 16: 196, 2008.

15. Jidovtseff B, Cordonnier, C, Binard, AS, Rulot, P, Maquet, D, Croisier, JL, Demoulin, C, and Crielaard, JM. Relevance of inertial fatigue test in sport applications. Isokinetics Exerc Sci16: 190, 2008

16. Jidovtseff B, Croisier, JL, Lhermerout, C, Serre, L, Sac, D, and Crielaard, JM. The concept of iso-inertial assessment: Reproducibility analysis and descriptive data. Isokinetics Exerc Sci 11: 53-62, 2006.

17 Jidovtseff B, Quièvre, J, Hanon, C, and Crielaard, JM. Les profils musculaires inertiels permettent une définition plus précise des charges d'entraînement. Sci Sports 24: 91-96, 2009.

18. Kraemer, WJ and Fleck, SJ. Strength Training for Young Athletes (2nd ed.). Champaign, IL: Human Kinetics, 2005.

19. Kraemer, WJ and Fry, AC. Strength testing: Development and evaluation of methodology. In: Physiological Assessment of Human Fitness. Maud, J and Foster, C, eds. Champaign, IL: Human Kinetics, 1995. pp. 115-138.

20. Madsen, N and McLaughlin, T. Kinematic Factors Influencing Performance and Injury Risk in the Bench Press Exercise. Med Sci Sports Exerc 16: 376-381, 1984

21. Mayhew, JL, Prinster, JL, Ware, JS, Zimmer, DL, Arabas, JR, and Bemben, MG. Muscular Endurance Repetitions to Predict Bench Press Strength in Men of Different Training Levels. J Sports Med Phys Fitness 35: 108-113, 1995.

22. Pereira, MI and Gomes, PS. Muscular strength and endurance tests: Reliability and prediction of one repetition maximum-Review and new evidences. Revista Brasileira de Medicina do Esporte 9: 336-46, 2003.

23. Pollock, ML and Evans, WJ. Resistance training for health and disease: Introduction. Med Sci Sports Exerc31: 10-11, 1999.

24. Ware, J, Clemens, C, Mayhew, J, and Johnston, T. Muscular endurance repetitions to predict bench press and squat strength in college football players. J Strength Cond Res 9: 99-103, 1995. 